\title{
Nocturnal Restless Arms and Face Improved by Dopamine Agonist: Is It a Variant of Restless Legs Syndrome?
}

\author{
Dae-seop Shin ${ }^{1}$, Seung Cheol Lee ${ }^{1}$, Soo A Kim² ${ }^{2}$ Hyung Ki Kim ${ }^{3}$, Kwang Ik Yang ${ }^{1}$ \\ ${ }^{1}$ Sleep Disorders Center, Departments of Neurology, ${ }^{2}$ Physical Medicine and Rehabilitation, and ${ }^{3}$ Clinical Pharmacology, \\ Soonchunhyang University of College of Medicine, Cheonan Hospital, Cheonan, Korea
}

Received May 12, 2017

Revised June 16, 2017

Accepted June 16, 2017

Address for correspondence

Kwang Ik Yang, MD, PhD

Sleep Disorders Center,

Department of Neurology,

Soonchunhyang University

College of Medicine,

Cheonan Hospital,

31 Soonchunhyang 6-gil,

Dongnam-gu,

Cheonan 31151, Korea

Tel: $+82-41-570-2290$

Fax: +82-41-592-3810

E-mail: neurofan@schmc.ac.kr
The symptoms of restless legs syndrome (RLS) usually involve legs and can extend to other body parts. However, isolated body parts other than leg have rarely been involved. A 32-year-old woman had abnormal sensations of both forearms and face during night, which caused a difficulty falling in sleep. The symptoms were relieved by shaking and rubbing. She experienced a clear benefit from pramipexole, but a recurrence of restlessness was followed in both arms subsequent to pramipexole withdrawal. Finally, she had been prescribed a dose of $0.375 \mathrm{mg}$, which was well tolerated for 6 years. We report a case of nocturnal restless arms and face without leg involvement, which was like RLS in terms of clinical criteria except lesion site.

J Sleep Med 2017;14(1):43-45
Restless legs syndrome (RLS) is a sensorimotor disease characterized by abnormal sensation in legs and motor restlessness that often has a profound impact on sleep. ${ }^{1}$ Essential diagnostic criteria include 1) an urge to move the legs usually accompanied or caused by uncomfortable and unpleasant sensations in the legs; 2) symptoms begin or worsen during periods of rest or inactivity such as lying or sitting; 3) symptoms are partially or totally relieved by movement such as walking or stretching; 4) symptoms are worse in the evening or night than during the day; 5) the above is not primarily explained by other medical or a behavioral condition like myalgia, leg edema and positional discomfort. ${ }^{1}$

RLS is treatable condition that generally responds well to dopaminergic agent. And the non-ergot dopamine agonists such as pramipexole are effective in the treatment of RLS. Although the symptoms of RLS usually appear in the legs first, extension to other body parts is relatively frequent. However, isolated body parts other than the legs have rarely been involved. We report a case of nocturnal restless arms and face

This is an Open Access article distributed under the terms of the Creative Commons Attribution Non-Commercial License (http://creativecommons.org/licenses/by-nc/4.0) which permits unrestricted non-commercial use, distribution, and reproduction in any medium, provided the original work is properly cited. without leg involvement, which was improved by dopamine agonist.

\section{Case Report}

A 32-year-old woman presented for evaluation with uncomfortable sensations in the forearms and lower face, which she had experienced for several months (Fig. 1). She reported her sensations as tingling, burning, itching and sometimes said she feels electrical currents are passing or worms are moving. These abnormal sensations appeared during the periods of inactivity such as lying posture and worsened in the evening and/or at night, resulting in difficulty in falling asleep due to an urge to move upper limbs at rest. These symptoms also occurred while sitting in a chair for 3-4 hours during the day. The symptoms were relieved by shaking and rubbing her forearms and face. She did not complain of leg cramp or muscle soreness, and her symptoms did not improve with simple posture changes. Although the symptoms were limited to forearms and lower face, they met diagnostic criteria of International RLS Study Group (IRLSSG). ${ }^{1}$ Her past medical history was unremarkable. She was not taking any regular medications. Skin 


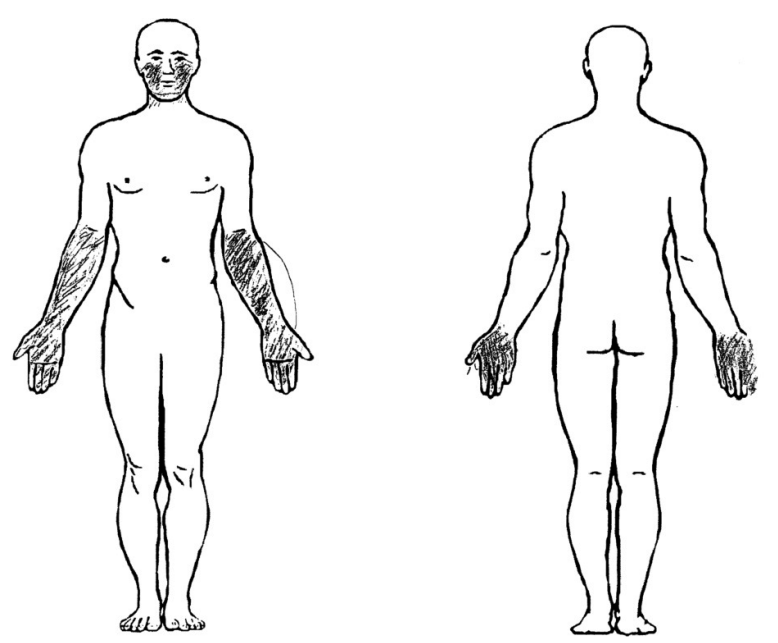

Figure 1. Distribution of sensory symptoms by patient's self drawing. Sensory symptoms are limited to the forearms, hands, and lower face.

lesions, leg edema or other focal neurological signs were absent. She reported no a family history of RLS. Her serum ferritin level was $169 \mathrm{ng} / \mathrm{mL}$ (13-150), hemoglobin was $13.9 \mathrm{~g} / \mathrm{dL}$ (12.0-16.0), total iron binding capacity was $284 \mu \mathrm{g} / \mathrm{dL}$ (254$437)$ and iron was $122 \mu \mathrm{g} / \mathrm{dL}$ (43-186). All levels were in normal range. And the results of her other blood chemistry tests including thyroid function were unremarkable. A nerve conduction study did not show any evidence of neuropathy. Initially, $0.125 \mathrm{mg}$ of pramipexole was started and her nocturnal restless symptoms in the forearms and face showed much improvement after a gradual titration to $0.375 \mathrm{mg}$ over $4-5$ months (International RLS Severity Scale 30 to 2). She experienced a clear benefit from pramipexole, but a recurrence of restlessness was followed in both arms subsequent to pramipexole withdrawal. The symptoms were relieved again when the medication was re-administered. Her nocturnal symptom fluctuated with multiple instances of decrease and increase in pramipexole administration. Her symptoms have been well responded to pramipexole. Finally, she had been prescribed a dose of $0.375 \mathrm{mg}$, which was well tolerated for 6 years.

\section{Discussion}

Our patient met the major criteria of RLS except the involved body part and responded to treatment with a dopamine agonist. Symptoms did not extend to other body parts beyond the arms and face. Involvement of other body parts along with the legs in RLS is relatively frequent. ${ }^{2}$ Although the most frequently involved areas are the arms, abnormal sensation could accompany anywhere in the body including the trunk, hips, shoulder, neck, genitals, and face. ${ }^{2}$ In addition, most of them usually occurred in patients with severe RLS or late course of the disease. However, isolated body parts other than the leg like our case have rarely been involved. ${ }^{3,4}$ A previous report indicated that the nocturnal unpleasant sensations were first localized to the arms and responded to a dopamine agonist, and then extended to the legs over a few years. ${ }^{3}$ In contrast, in our patient, the sensations did not extend to the leg for 6 years. Other patients have experienced difficulty in maintaining sleep due to abnormal sensations, which were localized to only abdomen. ${ }^{4}$ They also responded well to dopamine agonists, similar to our case.

A study investigated topography sensory symptoms of RLS based on clinical information but none of existing theories can explain why RLS symptoms mostly begin in the lower limbs, and why, in some cases, the leg symptoms extend to other body parts. It is hypothesized that pathophysiology of RLS intimately involves a dysfunction of dorsoposterior hypothalamic dopaminergic A11 cell group with descending pathways that target preganglionic sympathetic neurons, dorsal horn regions, interneurons, and somatic motor neurons, and a possible dysregulation in the excitability of the spinal cord networks. ${ }^{5}$ The loss of A11 dopamine modulation of the dorsal horn could result in disinhibition of sensory inputs, and thereby, favor the occurrence of abnormal visceral or muscle sensations. An abnormal sensation of this case was localized only in both hands and face, but not in legs, which could be inappropriate to compare with RLS because involvement of the legs is obligatory on IRLSSG criteria. Previous reports suggest the cases like this patient as variants of RLS and had good response to dopamine agonist.

The symptom in the present case was well controlled by a pramipexole $0.375 \mathrm{mg}$ during the 6-year follow up. The symptom fluctuations according to dosage variations could exclude the placebo effect. When the dosage was reduced, her symptoms aggravated. Proper dosage relieved her symptoms which could rule out the possibility of placebo effect.

In cases where abnormal sensations on body parts besides the legs are similar to RLS symptoms with circadian variation, that is, if the symptoms worsen in the evening and/or at night, we hypothesize possibility of a variant of RLS, even though further research should be conducted to clarify this by obtaining sufficient clinical data. We also carefully suggest that dysregulation of dopaminergic transmission from hypothalamus to spinal cord, which is topographically localized to arm and face, might be possible mechanism. And we recommend empirical treatment with a dopamine agonist. This case showed the similar symptoms like RLS except body parts that are involved in RLS and improved by dopamine agonist, pramipexole. 


\section{Acknowledgments}

The authors would like to thank Ms. Shinyoung Park who graduated from University of Southern California and majored in Communicaton and Journalism, and Biological Sciences there. She contributed to improve English writing.

\section{REFERENCES}

1. Allen RP, Picchietti DL, Garcia-Borreguero D, et al. Restless legs syndrome/Willis-Ekbom disease diagnostic criteria: updated International Restless Legs Syndrome Study Group (IRLSSG) consensus criteria--his- tory, rationale, description, and significance. Sleep Med 2014;15:860873.

2. Koo YS, Lee GT, Lee SY, Cho YW, Jung KY. Topography of sensory symptoms in patients with drug-naïve restless legs syndrome. Sleep Med 2013;14:1369-1374.

3. Horvath J, Landis T, Burkhard PR. Restless arms. Lancet 2008;371:530.

4. Pérez-Díaz H, Iranzo A, Rye DB, Santamaría J. Restless abdomen: a phenotypic variant of restless legs syndrome. Neurology 2011;77:1283-1286.

5. Clemens S, Rye D, Hochman S. Restless legs syndrome: revisiting the dopamine hypothesis from the spinal cord perspective. Neurology 2006;67:125-130. 